DOI: $10.1515 /$ hssr -2016-0015

\title{
Symmetrical Geometry of Flowers in Art and Nature "The Triumphal Entry of Jesus into Jerusalem"
}

Cristian Ungureanu

University of Fine Arts, Iasi, Romania*

\begin{abstract}
The aim of our study is to highlight the obvious similarities that exist between the organizational structures of the biological world, particularly in terms of the number and distribution of the petals on flower and the geometric configurations used by the great masters of European painting, both in the East but also in the West, in order to elaborate the compositional framework of paintings and icons. Taking into consideration the symbolic connotations concerning the field of biology (the primary focus this article), we chose as a case study the Russian icon from the $14^{\text {th }}$ century entitled "The Triumphal Entry of Jesus into Jerusalem", exhibited and venerated across the orthodox world on Palm Sunday. Starting with the circle of the aureole of Jesus, we have marked the harmonic series of concentric circles that are distributed across the nodal points of some regular polygons with seven and nine sides, which make reference to the ergonomic formulas of distribution of the petals on a flower and highlight the fact that the beauty of the natural world was and still is the main source of inspiration in the world of arts.
\end{abstract}

Keywords

Symmetry, Flowers, Nature, Art, Composition

\footnotetext{
"Faculty of Visual Arts and Design, "George Enescu" University of Fine Arts, 189, Sărărie St., Iaşi 700451, Romania; cn_ungureanu@yahoo.com
} 


\section{Introduction}

From a transdisciplinary perspective and through an extensive synthesis process of the data from different fields of investigation, the aspects concerning the ways to harmonically order the live matter from the natural world can be found in the mathematical theories concerning symmetry and the dynamics of harmonic sequences (Fibonacci) or the role of special irrational numbers $(\mathrm{Pi}=3,14159 .$. , Phi $=1,618033 .$.$) in the world of visual$ arts, as a source of inspiration for artists in all fields, eras and artistic currents who have marked the history of arts. These great models of harmonic ordering based of symmetries (or the ordered breaking of symmetries) are found to be present in the mineral world. These are also present both at a microscopic scale, at the level of the smallest identifiable particles through the use of nanotechnology, but also at the scale of the observable cosmos.

The icon "The Triumphal Entry of Jesus into Jerusalem", which is the subject of our case study, was painted by an anonymous monk, in $14^{\text {th }}$ century Russia. The knowledge regarding the science of geometric construction which the Eastern masters passed down through the iconographical schools located around the byzantine monasteries, is the same as the practice of western masters, and it is known that the origins of these methods, as far as the European artistic space is concerned, come from the spiritual and cultural centre of the Byzantine empire, Constantinople.

The general concept concerning the development of an iconographic model was that of generating certain rising harmonic rhythms, focused on a main point of interest, similar to the models of growth of living organisms. This nucleus was marked with a clearly stated clue and it was thought to have the power to generate and transmit to the conscience of the thinker the entire geometric and symbolic frame-work of the work of art. The knowledge and the systematic study of these constructive methods was lost/abandoned once the first signs of modernity appeared and the traditional forms of artistic learning (workshops and schools that were governed by a true master) were replaced with the art academies and individual workshops of the artists. The overall aim of the studies that we undertake is to extract and classify the defining data concerning this mathematical/geometrical language, filled with a multitude of symbolic 
nuances concerning each of the subjects in question but also to establish a broader transdisciplinary perspective, while highlighting the connections that exist between a practice that is specific to the art world and the other fields of knowledge, using modern investigative methods. It is well known that the beauty of the natural world, especially the one of plants and flowers, was a permanent source of inspiration to artists from all eras, from the painted walls of the caves in Altamira, to the most extravagant multimedia installations of the major festivals and contemporary art biennials. A partial knowledge concerning the way of thinking and the practical methods that were used by the old masters constitutes an impediment to such an investigation, but the interaction with different fields and branches of the digital contemporary culture can be a real step forward in the process of unveiling the conceptual complexities of the old art, and, perhaps, a possibility to overcome the identity crisis that the contemporary art finds itself in with the official ending of the cycle of modernity and the entry into the post-modern era.

The growth and organization models of plants and flowers shows the existence of certain preferences towards a polygonal order which gravitates, predominantly (notably and surprisingly), towards the numbers that make up the Fibonacci sequence $(1,3,5,8,13,21, \ldots)$. But, in equal measure, other numbers, including even numbers and the ones that generate symmetrical structures can be found as well. The problem/subject of the symmetry is one of the most relevant, both in the field biology but also in the visual and other arts.

\section{Methods}

The only systematic studies concerning the constructive methods of the artists from the past and the knowledge of the principles of organization and growth of the plant world are the international study of Matila Ghyka (1998) and the study of geometry of painting done by the French Charles Bouleau (1979), which was centered exclusively on structures and linear framing and on the proportions of the rectangles frequently used by the great artists of the previous eras, systematically neglecting the edifying aspect of circular and radiant structures of the harmonic and symmetrical compositional formations that derive from these, which our studies and documents (preparatory sketches of the paintings or the pictorial evidence 
of the analyzed works) identify as having a precise and determining role in establishing the configuration of paintings and icons.

As mentioned, symmetry is one of the most widespread aspects both in the natural world but also in visual arts, in architecture, in different fields of science, a theme that has preoccupied and fascinated humanity ever since prehistoric times. The oldest cave drawings or statues of the Paleolithic cultures are full of examples of visual decorative structures based on symmetrical configurations. The human mind is, undoubtedly, fascinated by symmetry, no matter where it is found and it can be said that this fact is a consequence of the symmetrical nature of the human body, a true spontaneous reflex towards the similarity of our own bipolarity.

Ever since antiquity, scholars, mystics and philosophers, have theorized over the various aspects and forms of manifestation of the symmetries. The old ones insisted on the symmetrical mirroring between the parts of an ensemble and its integral configuration, focusing on the problem of the rhythm that this perspective on the symmetry generates. The modern ones, from the Renaissance on, have started to lose interest and to forget the ways of understanding these concepts regarding symmetry, focusing on the immediate meanings of the concept of symmetry, the one concerning the identity of the halves pertaining to whole or of a certain phenomenon, and for the majority of our contemporaries, symmetry only means equal or identical. Interesting to note is that even a small deviation from a perfect symmetry from a mathematical point of view, is just as wanted and fascinating for the unending mechanisms of the human mind and soul. As British physicist Ian Stewart argues in his book Nature's numbers, even this temptation and fascination for the breaking of perfect symmetries is a phenomenon that is just as widespread in nature as well. Moreover, this deviation from the norm is the engine of natural evolution, generating the variation of species and the possibilities of adaptation to new environmental, natural and social factors. However, the great French mathematician Pierre Curie was the one who, together with his wife, discovered radioactivity and stated the general principle according to which "the effects are just as symmetric as their causes" (Steward, 2010: 87). It is noteworthy that, in practice, almost all the symmetrical forms from nature are less symmetrical than the causes that generate them. Modern physics has studied closely this phenomenon and called it "spontaneous symmetry 
breaking". We will follow several directions of this scientific study, in order to highlight "the symmetry" of the interest that the artists from all ages showed towards the multiple aspects of the symmetry found in the natural world but also in the plains of reality that are not seen, the meta-physical, aspects which inspired and fascinated to such an extent that they ended up being classified as norms and principles of organization of the bi and threedimensional space of the works of art.

The bipolar symmetry, very common in the natural world, presupposes the establishment of a law of mirroring, a doubling and a movement of components of one of the parts towards its opposite, in such a way that no changes occur, and the reflection is achieved through the use of an axis of symmetry, arranged perpendicular to the axis that connects the centers of the two symmetrical parts. As a general rule, it can be affirmed that symmetry is any transformation of an object or system, that keeps it invariant and without change. There are classifications of different types of symmetries, depending on the law of construction or induced movement. The most common are the reflections, rotations and translations. Reflections require the existence of an axis or of a plane of symmetry and determines that the left part must be the reverse of the right one, the rotations that generate symmetrical forms require the existence of a point or axis of symmetry, around which an object or an ensemble is rotated while keeping its original properties, like a flower with a random number of petals that are arranged at equal distances from one another and are rotated around the center. The translation or displacement requires generating a matrixtype system in which a component of the system, which is made up of identical pieces, can perform a vertical, horizontal or diagonal movement, without changing the defining qualities of the ensemble. The best example of this type of symmetry that is found in nature is the honeycomb.

The hexagonal structure of the honeycomb is most often seen as a pattern of compositional organization of a pictorial work of art and can be found, either as a linear form of the hexagon, or as a symmetrical and circular configuration of the "flower of life", a well-known constructive and symbolic formula, found in all the important cultures and civilizations of the world. However, in the case study chosen by us, the icon "The Triumphal Entry of Jesus into Jerusalem", which is in a direct symbolic relation with the palm tree, is conditioned by the presence of two 
polygonal and circular configurations originating from the numbers seven and nine. "These introduce a certain type of contemplation of the image and a possible trigger of certain resonances with the harmonic proportions enciphered in these schemes. It is more than likely that, at least during times of maximum stylistic purity or when the iconographical prototypes made their presence seen, the iconographical masters knew precisely the geometric schemes that corresponded to each of the scenes." (Ungureanu, 2006: 74).

Taking the halo around the head of Jesus as the main clue, we generated a structure of concentric circles with this circle as the base one, whose growth ratio is exactly the radius of the aureole. At the level of the diameter of the seventh circle we get the width of the painted rectangle of the icon (Fig.1). In order to overlay this circle on the vertical center line of the rectangle, a slight displacement towards the right of the other seven circles is necessary, just as the character of Jesus moves from left to right, an aspect that is suggested by the painting itself and its title. This slight asymmetry highlights the dynamic/cinematic character of the composition, both in the vertical and the horizontal plane, generating a sinusoidal trajectory which starts at the inferior part of the icon, goes through the left outline of Jesus' silhouette and continues its slanted trajectory towards the left of the tree that is near the walls of Jerusalem. The circular structure named by us as "The flower of seven", which pertains to the heptagon inscribed in the circle that is tangent to the painted rectangle of the icon, highlights through its "petals" the main standards of the pictorial composition and the radial trajectories that unite the geometric and conceptual center of the icon with the other areas of interest. (Fig. 2-019).

The same circle comprised of seven units/aureoles is subject to displacement of the structure of the "Sephirot tree" - the tree of life from Jewish doctrine. Lowered four times its radius, the mentioned circle indicates, through this structure, the possibilities of development of the vertical trajectories of the composition, marking the lower side of the painted rectangle of the icon but also the symbolic way in which the painting should be observed, the zigzag that unites the intersections of the four circles - the ten "Sephirots" of the "tree" (Fig.3). This trajectory, called "the lightning" in the Jewish tradition, starts from the 
center of the upper side of the rectangle and goes through the entire surface of the painting, indicating both the dynamics of the components but also the symbolic relation between these elements.

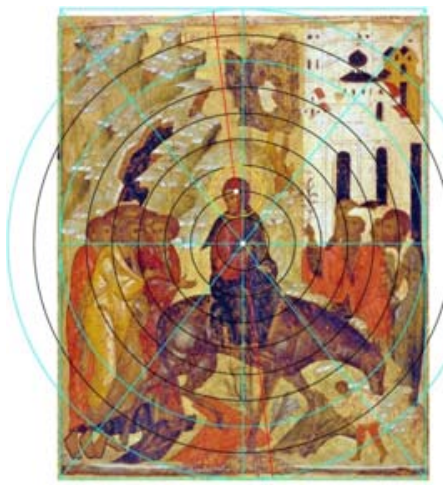

Fig. 1. The structure of nine concentric circles derived from multiplying the circle of the aureole

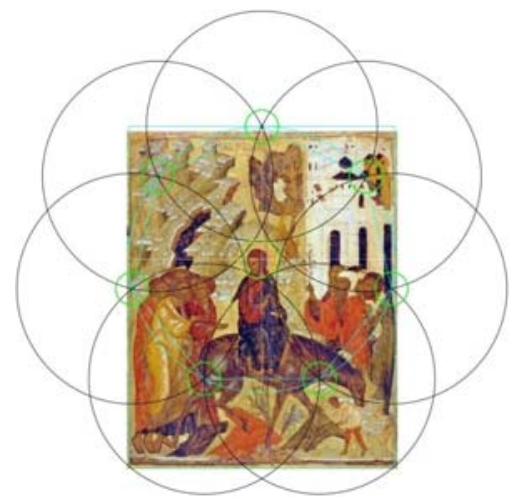

Fig. 2. Compositional structure of the "Flower of seven"

The circle comprised of nine units (the diameter of Jesus's aureole) equals the height of the compositional rectangle of the icon and when it is positioned in the center of the rectangle, the circle becomes tangent to the circle of the aureole of Jesus, amplifying the dynamic meaning of the composition both on the horizontal plane but also on the vertical one. The structure "Flowers of nine", which it develops, fulfils the same role of demarcation of each group of elements and characters that inhabit the surface of the icon, inscribing a radial structure through the intersection of the nine circles-"petals" (Fig.4). It is evident that the traditional symbolism (similar if not identical to the ones in the great religious and metaphysical traditions of humanity) is in close relation with the choice made by the icon master in structuring his composition, which is based on the properties of the numbers seven and nine. When it is inscribed in between the upper and lower poles, the circle of nine units appropriates the attributes of a ternary structure (Fig.4) and the networks of intersections indicates the manner of distortion and stylization of the characters and elements in the scene, generating the unmistakable particularities of the iconographical Byzantine painting, that of the 
inverse perspective and of a multi-spatiality.

The last part of our analysis of the geometric structure of the composition of the icon concerns the intrinsic properties of the chosen rectangle; this rectangle is atypical to Eastern iconography, having the ratio of $\mathrm{L} / 1=9 / 7-1,12$. It is inscribed in three large and equal vertical parts (Jerusalem - on the right, the tree - in the center and the mountain - on the right, constituting a symmetrical "mirrored" reflection of the "Holy Trinity" icon). The network of diagonals of the rectangles and the squares obtained by connecting the horizontal sides with the vertical ones, indicates the trajectories used to enhance the dynamic/cinematic character of the scene. In order to better illustrate how the geometric composition was generated and the relation between the stages of the geometric analysis of the icon, we have made a video animation entitled "The Triumphal Entry of Jesus into Jerusalem" (https://www.youtube.com/watch?v=Gi5HvTqElIU).

\section{Conclusion}

Our focus on this geometric analysis and on the symbolic highlights underlines the compositional complexity of this painting done in the Eastern ecclesiastical ambiance six centuries ago. The references to the field of biology, that can be found in the religious dimension of the subject, proves that observing the mathematical and harmonic rhythms and the generated aesthetic qualities of the natural world was always a subject of intense interest and inspiration for artists. 


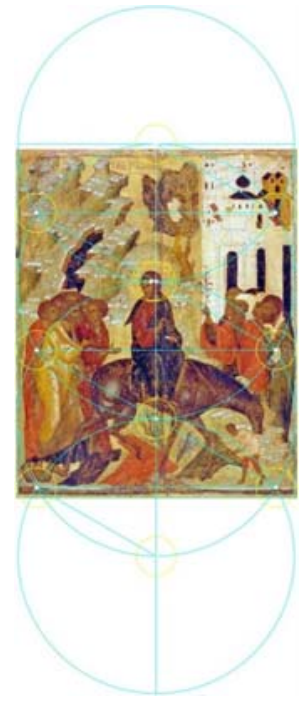

Fig. 3. The structure of the "Sephirot tree/the tree of life" generated by the seventh circle

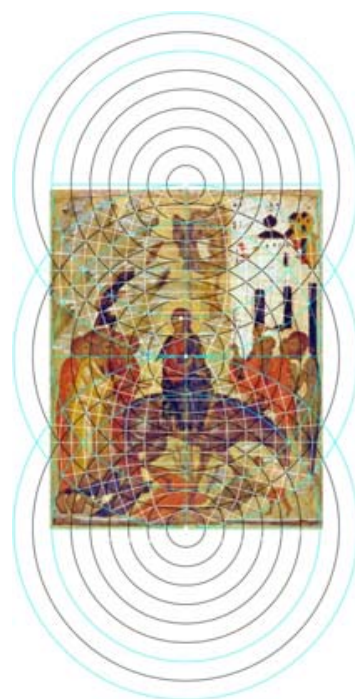

Fig. 5. Three-part compositional structure of the circles seven and nine

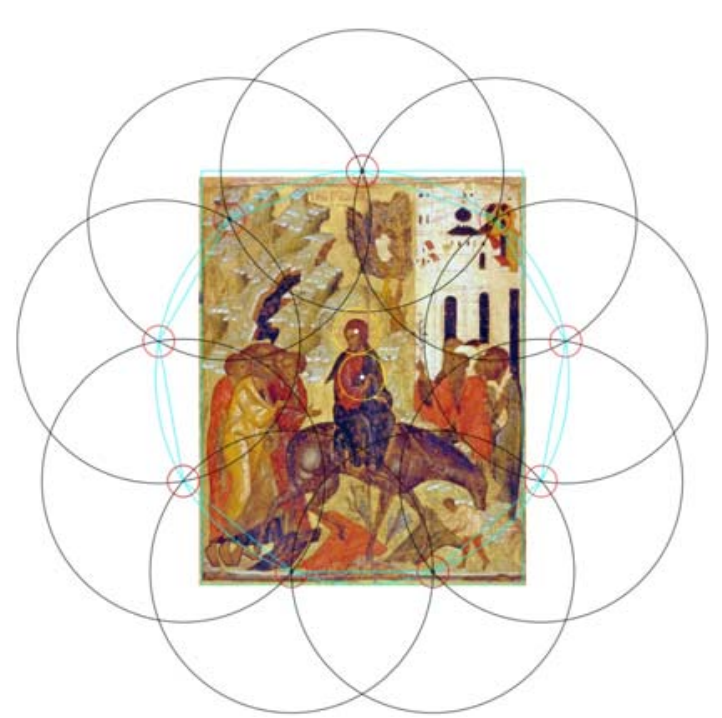

Fig. 4. Compositional structure of the "Flower of nine"

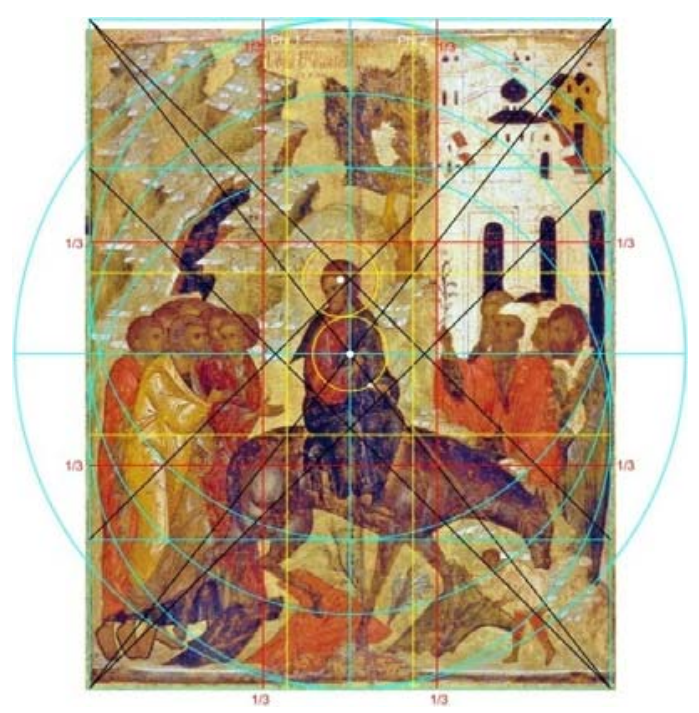

Fig. 6. Harmonic structure of the rectangle of the icon, $\mathrm{L} / \mathrm{l}=9 / 7=1,28$ 
The hypothesis of using circular geometric structures related to regular polygons ("the flowers" of different numbers and regular polygons) is based on many obvious examples such as the drawings of Leonardo da Vinci, the ones that have as subject polygons and regular polyhedrons, the well-known "flower of life" (the flower of six) or the recent Roman mosaics from the first century AD, discovered in Zeugma (Turkey), an archaeological site in which several mosaic compositions can be found under the form of medallions built on the bases "flowers" associated with the numbers six, eight and ten. We believe that this type of investigation could open a new perspective on the interdisciplinary and transdisciplinary studies, which also involves artistic projections of data that pertain to other fundamental fields of knowledge.

\section{References}

Bouleau, C. (1979). Geometria secreta a pictorilor. Bucuresti: Meridiane Publishing House.

Ghyka, M. (1998). Filosofia și mistica numărului. Bucuresti: Univers Enciclopedic Publishing House.

Stewart, I. (2010). Numerele naturii. Bucuresti: Humanitas Publishing House.

Ungureanu, C. (2006). Dialog între sferă și cub [Geometria secretă a picturii bizantine]. Iași: Artes Publishing House.

Bartos, J. (2005). Structuri compoziționale. Iaşi: Artes Publishing House. Bartos, J. (2009). Comporiția in pictură. Iași: Polirom Publishing House.

\section{Biographical note}

Cristian Ungureanu is a visual artist, $\mathrm{PhD}$ lecturer at "George Enescu" University of Fine Arts, Iasi, Romania and president of the Alumni Association of the same university. Member of the Editorial board of ANASTASIS, an international peerreviewed journal of Research in Medieval Culture and Art. He is the author of a large number of individual exhibitions, among which "Musique des sphères [La géométrie secrète de la peinture européenne]", held at the Romanian Cultural Institute in Paris (18 ian-18 feb 2016), and of art theory and artist books. 